\title{
Laparoscopy-Assisted Distal Gastrectomy with Jejunal Pouch Interposition
}

\author{
Seigo Kitano, MD, FACS and Norio Shiraishi, MD \\ Department of Surgery I, Oita University Faculty of Medicine, Oita, Japan
}

The incidence of early gastric cancer (EGC) has been increasing in Asian countries as a result of the development of diagnostic modalities such as endoscopic examination and barium meal examination. Currently in Japan, patients with EGC account for more than half of all patients with gastric cancer. More than $90 \%$ of patients with EGC can be cured only by curative gastrectomy. Therefore, in the treatment of EGC, not only complete removal of cancer cells but also minimal invasiveness and improvement of patient quality of life (QOL) after surgery are essential. ${ }^{1}$

Distal gastrectomy (DG) with Billroth-I (B-I) reconstruction is the most popular surgical treatment for distal EGC in Japan. Recently, this operation has begun to be performed minimally invasively with laparoscopy-assisted techniques. However, many patients who have undergone open DG or laparoscopy-assisted DG (LADG) have experienced postgastrectomy syndrome, which involves efflux esophagitis, dumping syndrome, and loss of appetite. To prevent postgastrectomy syndrome after DG or LADG, several modified gastrectomy techniques have been developed: pylorus-preserving gastrectomy (PPG), vagal nerve-conserving gastrectomy, and gastrectomy with jejunal pouch interposition (JPI), among others. ${ }^{2}$ In this issue of the Annals, Junhyun et al. report the technical safety of LADG with JPI and the better long-term QOL experienced by patients undergoing this operation. ${ }^{3}$

The techniques of LADG with JPI, and especially the procedure for forming the jejunal pouch, are more complex than LADG with $\mathrm{B}-\mathrm{I}$ reconstruction. In this procedure, creation of the jejunal pouch is performed under minilaparotomy in a manner similar to that under open surgery. Although autosuturing machines are used to simplify the

(C) Society of Surgical Oncology 2010

Published Online: 5 May 2010

S. Kitano, MD, FACS

e-mail: kitano@med.oita-u.ac.jp techniques of jejunal pouch creation, there are several technical risks including ischemic change of the pouch, bleeding from the sutured edge, and ischemic change at the site of the pouch-duodenal anastomosis. To overcome these problems, several improved techniques have been devised: jejunal J-shaped pouch, short-length jejunal pouch, and use of a surgical stapler with a long slender shaft and three rows of staples (white cartridge). The study by Junhyun et al. evaluated the outcomes of LADG with a $10-\mathrm{cm} \mathrm{U-}$ shaped pouch made with a linear stapler with two rows of staples. Although the incidence of postoperative complications was not high, 2 of 25 patients had intra-abdominal bleeding. Thus, it is necessary to establish safe techniques for the performance of LADG with JPI.

LADG with JPI was developed to provide patients with EGC advantages such as early recovery after surgery and improvement in postoperative QOL. In their article, Junhyun et al. report good QOL in patients who underwent LADG with JPI compared with those who underwent Billroth-II (B-II) reconstruction. Few reports address postoperative QOL after DG with JPI because of several problems related to research on patient QOL after gastrectomy.

First, most important is determining what kind of postDG reconstruction method should be used as a control in the study of patient QOL. Patient QOL after gastrectomy primarily depends on the reconstruction method. Theoretically, dietary volume, systems to prevent efflux, and speed of digestion and movement of food into the duodenum may affect patient QOL. The aims of JPI are to increase dietary volume and decrease the symptoms of efflux. Junhyun et al. compared postoperative QOL after JPI reconstruction with that after B-II reconstruction. Although B-II reconstruction is the most popular method used in China and Korea, a comparison study between LADG with and without JPI should be performed to clarify the effect of JIP on postoperative QOL. A number of reconstruction 
methods, such as B-I, B-II, Roux-en-Y, pylorus-preserving gastrectomy with JPI, and vagus nerve-preserving DG with JPI are available. In addition to these reconstruction methods, many variations of JIP have also been reported. ${ }^{4}$ The length and volume of JPI, the shape of the JPI, and the volume of remnant stomach may affect postoperative QOL. Because DG with JPI reconstruction is derived from DG with B-I reconstruction and not B-II reconstruction, B-I reconstruction should be use as a control to clarify the effect of JPI on postoperative QOL.

Second, how to obtain objective results from a subjective patient questionnaire is important. Regrettably, no standard methods presently exist to adequately evaluate patient QOL after surgery. As well, statistical analysis of a large number of samples will be necessary to obtain objective results.

The number of patients with EGC who are undergoing curative gastrectomy and who are suffering from gastrectomy syndrome has been increasing. The development of gastrectomy with better methods of reconstruction than those currently at hand has long been awaited to improve postoperative patients' QOL. Randomized, multicenter control studies to clarify the best method of gastrectomy and reconstruction among the current methods from the viewpoint of postoperative patient QOL are necessary.

\section{REFERENCES}

1. Kitano S, Shiraishi N. Minimally invasive surgery for gastric tumors. Surg Clin N Am. 2005;85:151-64.

2. Mori Y, Arita T, Shimoda K, Yasuda K, Matsui Y. Inomata M, et al. Jejunal interposition to prevent postgastrectomy syndromes. Br J Surg. 2000;87:1576-9.

3. Lee J, Hoon H, Wook K. Improved long-term quality of life in patients with laparoscopy-assisted distal gastrectomy with jejunal pouch interposition for early gastric cancer. Ann Surg Oncol. 2010. doi:10.1245/s10434-010-1095-z.

4. Liedman B, Hugosson I, Lundell L. Treatment of devastating postgastrectomy symptom: the potential role of jejunal pouch reconstruction. Dig Dis. 2001;18:218-21. 